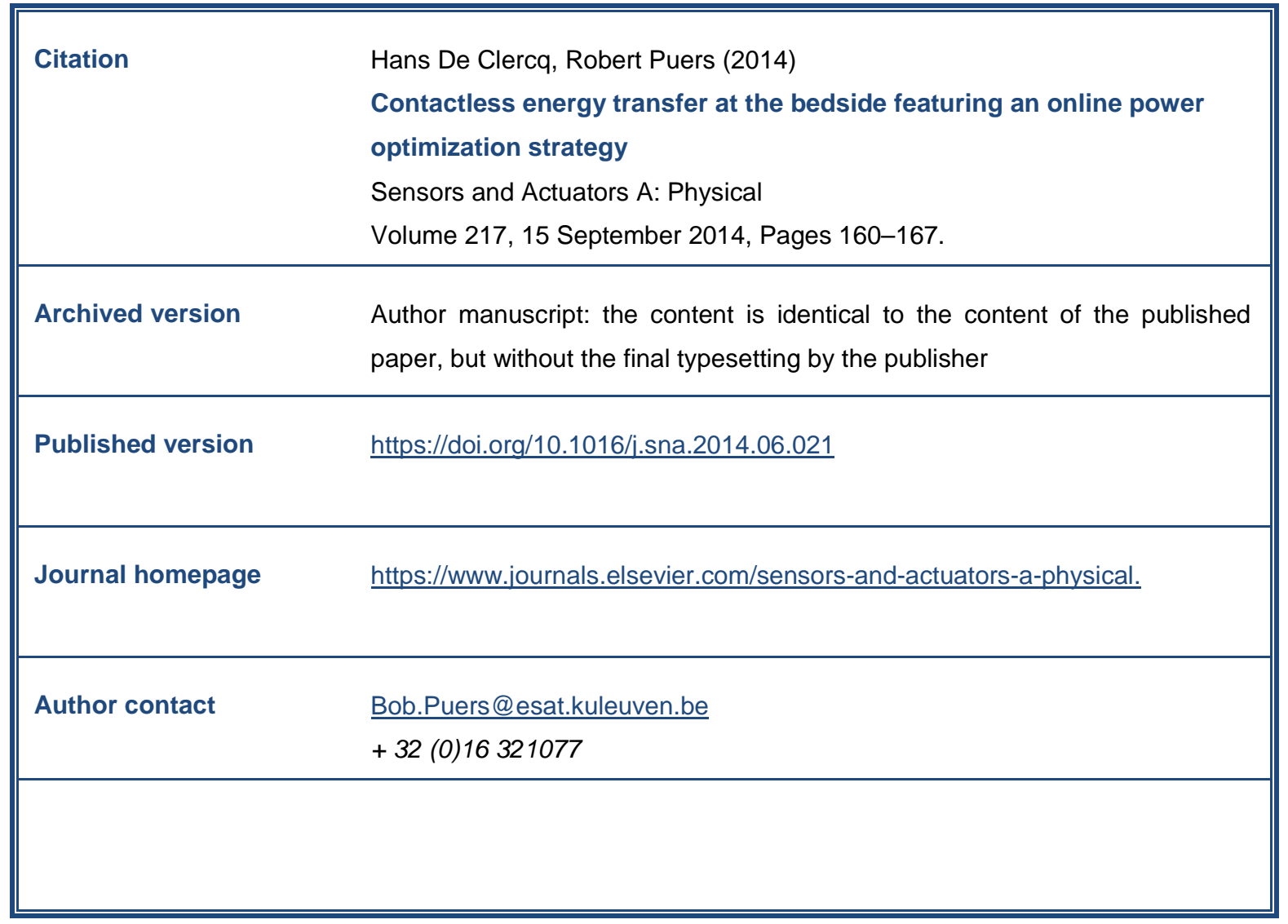

(article begins on next page) 


\title{
Contactless energy transfer at the bedside featuring an online power optimization strategy
}

\author{
Hans De Clercq*, Robert Puers
}

Katholieke Universiteit Leuven, Departement Elektrotechniek, ESAT-MICAS, Kasteelpark Arenberg 10, 3000 Leuven, Belgium

\begin{abstract}
This paper introduces the principle of contactless energy transfer to wearable bedside electronics. As a proof of concept, a platform containing a matrix of magnetic energy transmitters has been developed and integrated into a mattress. This system is able to feed one or more wearable sensor nodes, attached to the patient, with the purpose of wirelessly recording vital signals over an extended period of time. Each of these power transmitters is designed to transfer up to $10 \mathrm{~mW}$ of power over a maximal distance of $300 \mathrm{~mm}$. Moreover, the platform embeds online optimization software to deal with vertical as well as horizontal sensor node movements. The position and orientation of each freely moving sensor node can be dynamically determined and a local magnetic field, with a matching radiated power level, can be selectively induced in the area where the sensor nodes are positioned. This approach therefore limits the radiation exposure to the patient to the minimum viable level. The prototype is successfully tested in a laboratory set-up.
\end{abstract}

\section{Introduction}

Emerging technologies such as cloud computing and the Internet of Things have made the world increasingly connected. Ubiquitous wearable devices are spanning an ever increasing range of applications. Such sensor networks are helping athletes to break records, patients to become more aware of their disorders, gamers to have a better virtual experience and employees to develop their careers [1].

The promising global impact of these technologies and the constant research progress in the field of vital signal acquisition promote clinical application of these devices [2-4]. Wearable health systems outshine classic, bulky bedside monitors and bypass wires that hamper the patient's freedom to move. This technology therefore greatly emphasizes an increase of comfort and facilitates long-term bio-signal acquisition. Moreover, these devices enable an effective rehabilitation period in a home environment. Home health is not only beneficial to the patient's well-being, but also stimulates cost-effectiveness in global health [5]. This promise of a revolutionized health care has made wearable electronics one of the most appealing areas in biomedical research [6-9].

Modern diagnostics, however, require long-term, high-quality data acquisition. Moreover, a multitude of algorithms for online bio-signal extraction and automatic pathology detection, are ready to be integrated in such a medical body sensor network but are in need for high performance, ergo they consume abundant energy [10-12]. On the other hand, the patient's comfort level is affected by the device's dimensions. Today the energy density level of commercially favorable lithium-ion batteries is typically limited to 0.75 $\mathrm{Wh} / \mathrm{cm}^{3}$ [13]. Innovative batteries that are expected to exceed these conventional lithium-ion batteries are attracting much attention in both the academic realm and industry. Recent research has indeed demonstrated energy density improvements up to $2.7 \mathrm{Wh} / \mathrm{cm}^{3}$ when using lithium-sulfur technology [14]. Although, because many practical issues with these technologies remain unsolved, it is expected that lithium-ion batteries will prevail over other batteries at least within a few decades [15]. Therefore, the use of battery-powered sensor networks in the field of medicine is hindered by a challenging trade-off between functionality, miniaturization and autonomy.

Inductive power transfer is a viable alternative to batteries to overcome this shortage of on-board 
available energy and is efficient enough to avert such a compromise. This technique was already introduced in the 1960s $[16,17]$ and has been widely used in the field of medicine to bias medical implants [18-20]. In case of implantable devices, this technique is accordingly referred to as transcutaneous energy transfer (TET). But also wearable electronics, used for bedside monitoring, could benefit from such a wireless magnetic link. In that case, we propose a system that consists of a fixed, base coil in the bed, and one or more remote monitoring units attached to the patient.

In contrast to transcutaneous energy transfer, which in most cases can be performed with a relatively high and fixed coupling factor, inductive powering at the bedside implies loosely coupled coils with significant variations due to patient and sensor movement [21].

In previous work, a life-sized clinical setup based on a Helmholtz configuration was devised to deal with similar coupling fluctuations [22-24]. This topology produces a magnetic field that is more uniform and stronger than a solenoid topology for the same excitation current [25]. The resulted field strength was proven to be homogenous within a constrained working volume of $0.18 \mathrm{~m}^{3}$ and to be big enough to power the sensor at every position and orientation within this space. Nevertheless, two potential complications, concerning the safety norms of human body exposure to alternating electro-magnetic fields, have arisen. Firstly, to cope with high coupling variations, such a magnetic link is typically optimized for a worst-case scenario, leading to excessive power consumption during nominal operation. Secondly, in this manner, the full upper body part is exposed to the induced magnetic field, which may conflict with the specific absorption rate standards $[26,27]$.

In the consumer market, similar energy transfer systems are rapidly gaining momentum. These contactless energy transfer (CET) platforms enable a convenient new way to charge mobile consumer products [28-30]. A popular choice in literature to increase the freedom of CET device placement and to allow the charging of several devices simultaneously, is by using multiple primary windings [31]. To increase the platforms efficiency, in some systems the primary coils can be individually activated so that only a few primary windings closest to the load device are turned on [32].

This resulted in the establishment of the Wireless Power Consortium and the introduction of the Qi standard for charging mobile electronic devices through magnetic induction [33]. Regulation of the output voltage is provided by a digital control loop where the power receiver communicates with the power transmitter and requests more or less power. The combination of the CET platform topology and the power regulation strategy, utilized by the Qi standard, could cope with the aforementioned obstacles; only a local magnetic field is induced in the area where the power receiver is positioned and excessive power consumption is reduced by regulating the voltage level dynamically.

Nevertheless, this standard has several shortcomings when applied to bedside patient monitoring. Firstly, the Qi standard is devised for a close and stable inter-coil spacing, which makes it possible to use backscatter modulation as a communication technique [21]. The loosely coupled coils, prone to contactless energy transfer at the bedside, makes inductive downlink communication infeasible. Secondly, in case of mobile device charging, we can assume that the inductive coupling will remain constant after an initialization phase. To deal with the aforementioned continuous coupling variations, the transferred power needs to be continuously adjusted. And lastly, this standard uses a relatively low operating frequency between $110 \mathrm{kHz}$ and $205 \mathrm{kHz}$. Increasing this frequency goes along with efficiency boosting and higher inter-coil distance endurance [34]. Moreover, the induced electromagnetic field diminishes and consequently patient safety increases. Faraday's law indeed states that for a higher frequency a similar electromotive force can be derived for a smaller magnetic flux.

This paper describes the required adjustments to the CET platform model in order to cope with wearable bedside electronics. As a proof of concept, a wireless energy transfer platform consisting of a matrix of 
identical base coils, integrated at the bedside, has been constructed. The implementation of the system and the measurement results will be discussed.

\section{Materials and methods}

The proposed contactless energy transfer platform consists of three parts. The first element is the targeted wearable sensor network. This system consists of several custom-made sensor nodes that are in charge of vital signal acquisition. The second component is a grid of inductive power transmitters, which are optimized to transfer abundant energy over a considerable distance. The third item is a centralized management unit that processes the gathered bio-signals and supervises the matrix of power transmitters. Fig. 1 illustrates a block-level schematic of this architecture.

\subsection{The remote wearable sensor node}

On one hand, a signal acquisition system is only as good as the data it delivers, on the other hand, these devices need to be made as small and unobtrusive as possible to enhance patient comfort. Therefore a custom-made sensor network for wearable bio-signal acquisition has been developed in house. The system architecture of each node is modular and consists of two circuit boards. The sensor interface circuit board contains a dedicated analog front-end, such as an amplifier to record biopotentials, an inertial measurement unit to measure patient movement or a pulse oximeter for photoplethysmography applications. The data and power circuit board subsumes the general-purpose data acquisition and power management blocks. Consequently, the nodes are easily adaptable for miscellaneous vital signs acquisition by tailoring the sensor interface circuit board. Both circuit boards also serve as battery contacts for a rechargeable coin cell battery of $10 \mathrm{mAh}$. The two electronic circuits are interconnected through the case wall as is explained in [35]. The nodes are updated with a more performance-rich microcontroller core (MSP430) to enable online signal processing. Also the radio band is altered to the $433 \mathrm{MHz}$ ISM band such that medical applications that work with implantable and swallowable systems can be addressed as well. The power consumption of each sensor node is less than $10 \mathrm{~mW}$ at a voltage of $3 \mathrm{~V}$. The module is surrounded by a helical coil that acts as the magnetic power link receiver and incorporates circuitry to rectify and measure the voltage over the coil. An assembled sensor node measures $5 \mathrm{~mm}$ high and has a diameter of $17 \mathrm{~mm}$. Fig. 2 shows the construction of such a remote wearable sensor node.

\subsection{The centralized data management unit}

The centralized data management unit carries out two distinct tasks. First of all, it is in charge of the physiological data. The data management unit communicates with a maximum of ten wearable sensor nodes through a $433 \mathrm{MHz}$ link. It gathers and processes the bio-signal data and it creates the possibility to online adjust data acquisition parameters such as the data rate, RF power, and specific sensor settings. Additionally, this unit can be connected to a computer via a USB interface for further offline signal processing and visualization purposes. Secondly, this main unit administers the wireless power link of the multiple power transmitters. It can address each inductive power transmitter individually via an SPIinterface. The MCU of the management unit exhibits the power optimization algorithm that adjusts the driver's output power, as well as the sensor tracking algorithm that determines the most fit power transmitters to establish magnetic energy transfer [36]. The power optimization strategy is detailed out in Section 2.5.

\subsection{The inductive power transmitter}

The paramount component of the proposed system is a matrix of eighteen identical pancake base coils integrated at the bedside. These spiral coils are arranged into a two-layer configuration as shown in Fig. 3. 
The coils in the bottom layer are overlapping with respect to the coils in the top layer. Such a topology can produce a local magnetic field at each position above its surface, such that the wearable sensor nodes can be placed randomly on top of the patient [37,38,31]. The coil matrix is embedded in a $600 \mathrm{~mm}$ by 450 mm medium-density fiberboard (MDF). To sustain a real pancake geometry, the coil is milled into the plate using a CNC milling machine. The coil has an outer diameter of $140 \mathrm{~mm}$, an inner diameter of 100 $\mathrm{mm}$ and contain ten Litz wire windings. Litz wire, a wire that consists of many thin wire strands, individually insulated and twisted, is designed to reduce the skin effect and proximity effect losses in conductors used at frequencies up to about $1 \mathrm{MHz}$ [39]. The average inductance of the coils measures $20.2 \mathrm{H}$. Reproducibility of our coil manufacturing process revealed an inductance deviation of $100 \mathrm{nH}$. This variability is due to the poor winding properties of the used wire. The geometric properties of the coils are carefully selected to obtain a trade-off between high coil quality, which improves the link efficiency, and a limited coil inductance, to reduce the voltages at the driver side [40]. To comply with the EMC regulations [41] and to increase the power link efficiency [23], the system is shielded by applying ferrite sheets (IRJ17) at the bottom [38,42].

Each coil is driven by a dedicated, high-efficient class E power amplifier [43], which is built in house based on off the shelf components. This power amplifier can efficiently drive a sinusoidal current through the base coil as will be discussed in Section 2.4. The electronics needed to adequately drive the power transmitter are gathered on a circular printed circuit board, which is placed in the middle of each inductor. The amplifier contains a power MOSFET (CSD18534), a power MOSFET driver (UCC27524) and a load network to shape the proper class E voltage and current waveforms. A local microcontroller (MSP430F2132) is used to steer the class E amplifier by generating a pulse width modulated signal with a frequency of $1 \mathrm{MHz}$ and a duty cycle of $25 \%$. Circuitry to regulate the radiated field strength is added as well. Fig. 4 depicts the circuit- level architecture of one of the wireless power drivers and Fig. 5 shows the realized platform.

Because this specific prototype is intended to be validated for longterm vital signal monitoring at the neonatal intensive care unit, it is designed to satisfy the anthropometry of newborns [44]. In most world nations, the total body length of newborns is between 35.6 and $50.8 \mathrm{~cm}$.

\subsection{Link realization}

The inductive power transfer mechanism can be maximized for given geometry and power requirements. Our in house developed optimization strategy of a loosely coupled magnetic power link can be subdivided into two parts [40]:

- The magnetic design: optimizing the link for given coil sizes, coil separation and frequency.

- The electronic design:tuning the additional circuitry to efficiently drive a considerable AC-current into the base coil.

The magnetic design implies the construction of a set of coils that optimally fits to the given load. The application-specific geometric aspects set an upper limit on the link efficiency that can be achieved. In our case, the base coils are limited to a mean radius of $60 \mathrm{~mm}$ and the radius of the remote solenoid inductor is $10 \mathrm{~mm}$, which is determined by the size of the sensor module. Furthermore, for this specific prototype a nominal inter-coil distance of $100 \mathrm{~mm}$ is appointed. This distance corresponds to the average abdominal height of newborns plus a small-sized mattress, which can be put on top of the coil matrix. These dimensions yield a coupling factor of a mere $0.34 \%$, which is experimentally verified using a twostep procedure [40]. With the given base inductor and remote load, the optimal remote coil parameters can then be derived by an iterative optimization process $[45,40,36]$. An inductance of $1.18 \mathrm{H}$ and a quality factor of 250 is found. The efficiency of the magnetic link, defined as the ratio of the useful power 
dissipated in the a.c. load at the remote side to the power put into the inductive link at the base side is then $27 \%$. The link power losses are mainly determined by the parasitic resistance of the base and remote coils.

For the electronic design, a class-E driver is selected to drive the base coil. In this topology, the power MOSFET operates as an on/off switch and the load network shapes the voltage and current waveforms to prevent simultaneous voltage over and current through the transistor. This driver exhibits high efficiencies even at high frequencies, due to zero turn-on voltage characteristics [43]. A duty cycle of $25 \%$ is chosen to minimize the required supply current. Spice simulations have shown a driver's efficiency of $97.87 \%$ in case of nominal inter-coil distance. The small losses are mainly due to the finite on resistance of the transistor.

A great advantage of loosely coupled coils is that coupling and load variations only cause voltage fluctuations at the secondary side; the class-E regime does not get detuned. To illustrate this, Fig. 6 depicts the Spice simulation results for a coil separation of $220 \mathrm{~mm}$. The simulations take the losses of the power transistor into account, the passive components, however, are represented by ideal elements. The top graph shows the driver's input signal, $\mathrm{V}_{\mathrm{GS}}$. The second graph shows the drain-to-source voltage, $\mathrm{V}_{\mathrm{DS}}$ , in black and the current through the transistor, $\mathrm{I}_{\mathrm{DS}}$, in red. The graph clearly indicates that the drain-tosource voltage is zero when the switch is open even when the coil separation is far from the nominal case. The last graph shows the voltage over the base coil in black and the voltage over the remote coil in red. In this case, the driver's supply voltage is $6.5 \mathrm{~V}$.

\subsection{Link optimization software}

Instead of creating a homogenous magnetic field within the working space of the remote system, the power transfer platform is able to dynamically determine the position and orientation of each freely moving sensor node and to selectively induce a local magnetic field with a corresponding radiated power level in the area where the sensor nodes are positioned. Doing so, our system epitomizes the power regulation strategy of the Qi standard. To realize this, the centralized data management unit features two link optimization algorithms. The complete optimization strategy is depicted in Fig. 7.

The sensor tracking algorithm deals with horizontal node displacement. During an initialization phase, the software toggles every base coil on and off subsequently and calculates which of the different coils needs to be activated to provide each sensor with optimal power. During run time, this procedure regularly restarts determining the best candidate, beginning with the adjoining transmitters. This link optimization strategy limits the body exposure to the area where the sensor nodes are positioned.

The power optimization algorithm adjusts the driver's output power to cope with coupling variations due to vertical node displacement, node tilting or axial inter-coil variations. This is done by altering the supply voltage of the active inductive power driver based on the induced voltage level at the sensor side. This feedback loop is controlled by a PI controller, which is tuned using the Ziegler-Nichols method [46]. The procedure permits to heuristically determine the optimal proportional and integral terms of the PI controller, by reproducing a step response signal. This is done by inducing an abrupt supply voltage change of $1 \mathrm{~V}$ at the driver side. The proportional and integral terms can then be derived from the corresponding supply voltage curve at the sensor node side. For the discussed prototype this results in the following PI controller equation:

$$
u(t)=K_{p} * e(t)+K_{i} * \int_{0}^{t} \mathrm{e}^{\tau} \mathrm{d} \tau
$$


where the proportional constant, $\mathrm{K}_{\mathrm{p}}$, is 0.959 , the integral constant, $\mathrm{K}_{\mathrm{i}}$ is 0.038 and where $\mathrm{e}(\mathrm{t})$ is the control error, given by the difference between the desired voltage level and the actual voltage level at the sensor node side. The resulted desired supply voltage, $\mathrm{u}(\mathrm{t})$, is sent to the microcontroller of the active power transmitter via an SPI interface. The actual supply voltage of the power driver can be regulated between $1.25 \mathrm{~V}$ and $20 \mathrm{~V}$ through an adjustable voltage regulator (LM317). The regulator's voltage level is approximated by:

$$
V_{\text {out }}=1.25 *\left(1+\frac{R_{A D 5292}}{R}\right) \text {, }
$$

where $\mathrm{R}$ is a resistance with a fixed value of $1 \mathrm{k}$ and $\mathrm{R}_{\mathrm{AD} 5292}$ is the resistance of a digital controllable potentiometer, AD5292, as shown in Fig. 4. This results in a minimal viable magnetic field intensity and a constant voltage level at the remote side.

Both algorithms are programmed into the microcontroller, incorporated in the centralized data management unit and are triggered with the same input value, the induced, rectified voltage at the remote side. The $433 \mathrm{MHz}-$ link, which is already conducting the medical data transfer, is used to administer the power feedback as well. This feedback loop is highlighted in red in Fig. 1.

\section{Results and discussions}

\subsection{The inductive power transmitter}

Fig. 8 shows the measurement results of the class E driver captured by a digital oscilloscope (TPS1154). The supply voltage during this measurement is kept constant at $6.5 \mathrm{~V}$. The top graph shows the driver's input signal, $\mathrm{V}_{\mathrm{GS}}$. A good agreement between this measurement and the ideal block wave, shown in Fig. 6 , is obtained by properly matching the drive voltage with the MOSFET being driven. The second graph shows the measured drain-to-source voltage, $\mathrm{V}_{\mathrm{DS}}$ together with the corresponding simulation result (dashed line). The deviation between simulation and measurement is caused by two deficiencies. Firstly, the class E regime is highly-sensitive to capacitance mismatch. While the simulations are calculated with a $0.1 \mathrm{pF}$ accuracy, the real capacitance variability exceeds $5 \%$. Secondly, the measured coil quality is a factor two lower than the calculated value. This is caused by contact resistance losses and eddy currents, which were not taken into account during simulation. The sub-optimal class E operation requires a higher peak voltage across the switch to produce a similar field strength [47]. The bottom graph shows the resulting base coil voltage. While in simulation a supply voltage of $6.5 \mathrm{~V}$ is enough to span $220 \mathrm{~mm}$, the real inter-coil distance is $190 \mathrm{~mm}$. Despite the substandard conditions, the driver's efficiency still exceeds $90 \%$. During nominal operation the class E efficiency measures $92 \%$, the total efficiency measures $21 \%$. This power link efficiency does not consider the power losses due to the microcontroller and the MOSFET driver.

\subsection{The power optimization algorithm}

The power optimization algorithm is compared to the classical approach in which the magnetic link is designed to cope with a worst case scenario. As an example, a maximal inter-coil distance of $250 \mathrm{~mm}$ and a received voltage level of $2 \mathrm{~V}$ is selected. Fig. 9 shows the results of both measurements. The top graph shows the driver's supply voltage. The second graph shows the received voltages at the secondary side. For the classical approach, the feed of the power amplifier is kept constant to $10 \mathrm{~V}$ such that a voltage 
transfer of $2 \mathrm{~V}$ at maximal coil separation is guaranteed. Introducing inter-coil distance variations between $50 \mathrm{~mm}$ and $300 \mathrm{~mm}$ results in extremely high voltage fluctuations at the remote side. It is clear that in the nominal case, an abundant voltage level is received, which inevitably corresponds to a higher magnetic field intensity. Moreover, additional voltage protection circuitry needs to be incorporated at the sensor side. When the power optimization method is applied, the driver's supply voltage is automatically adjusted between $1.25 \mathrm{~V}$ and $20 \mathrm{~V}$. Consequently, the magnetic field intensity is optimized and the voltage level at the remote side is kept constant. The optimization strategy is limited in two directions. The upper limit is specified by the characteristics of the used components. The maximal $\mathrm{V}_{\mathrm{DS}}$ is set to $60 \mathrm{~V}$. The minimal inter-coil distance is limited by the behavior of the class E power amplifier. This is illustrated in the bottom graph of Fig. 9. The total efficiency, presented by the dashed line is maximal around a coil separation of $100 \mathrm{~mm}$. The link efficiency (red) diminishes when the distance increases, therefore a higher supply voltage is needed. When the distance decreases, the class E efficiency becomes the bottle neck. The higher coupling damages the class E regime.

\section{Conclusions}

The presented research has introduced inductive energy transfer to longterm vital signal acquisition at the bedside. A specific prototype that consists of a matrix of transmitting coils, covering an area of $0.27 \mathrm{~m}^{2}$, has been developed with the aim of powering a neonatal body sensor network. A software algorithm, which can automatically assign the most efficient power transmitter to bias a freely moving remote sensor node, has been integrated into the system. Experiments have proven the system's aptitude to deal with online horizontal as well as vertical sensor movement. Effort has been put in the design, simulation and implementation of a highly efficient, loosely coupled inductive link. The system makes it possible to provide $10 \mathrm{~mW}$ of power to a remote node at an intercoil distance between $50 \mathrm{~mm}$ and $300 \mathrm{~mm}$. Future work will focus on further characterization of inductive power transfer at the bedside and on patient safety. First of all, the near-field electrical and magnetic radiation will be measured and compared to the specific absorption rate safety standards. Secondly, integration tests are scheduled in the near future to validate the system in a clinical environment and to rule out electromagnetic interference with other potential medical equipment.

\section{Acknowledgments}

This research was partially funded by the Research Foundation Flanders (FWO-V). We would also like to acknowledge the Institute of the Promotion of Innovation through Science and Technology in Flanders for the doctoral fellowship granted to H. De Clercq. ESAT-MICAS is supported by the Hercules foundation for heavy equipment.

\section{References}

[1] Rack Space, The Human Cloud: Wearable Technology from Novelty to Production, Rack Space, 2013,

http://www.rackspace.co.uk/sites/default/files/ whitepapers/The Human Cloud - June 2013.pdf (accessed: 29.07.14).

[2] H. Alemdar, C. Ersoy, Wireless sensor networks for healthcare: a survey, Com- put. Netw. 54 (2010) 2688-2710.

[3] P.Binkley,Predictingthepotentialofwearabletechnology,IEEEEng.Med.Biol. 22 (2003) 23-27.

[4] P. Bonato, Wearable sensors/systems and their impact on biomedical engineering, IEEE Eng. Med. Biol. 22 (2003) 18-20.

[5] A. Milenkovic, C. Otto, E. Jovanov, Wireless sensor networks for personal health monitoring: issues and an implementation, Comput. Commun. 29 (2006) 2521-2533.

[6] M. Chan, D. Estve, J. Fourniols, C. Escriba, E. Campo, Smart wearable systems: current status and future challenges, Artif. Intell. Med. 56 (2012) 137-156. [7] J. Rabaey, J. Ammer, T. Karalar, S. Li, B. Otis, M. Sheets, T. Tuan, PicoRadios for wireless sensor networks: the next challenge in ultra-low power design IEEE International Solid-State Circuits Conference, 2002. Digest of Technical Papers. ISSCC, vol. 1, 2002, 


\section{Submitted to Sensors\&Actuators, June 2014}

pp. 200-201.

[8] M. Sawan, M. Salam, J. Le Lan, A. Kassab, S. Gelinas, P. Vannasing, F. Lesage, M. Lassonde, D. Nguyen, Wireless recording systems: from noninvasive EEG-NIRS to invasive EEG devices, IEEE Trans. Biomed. Circuits Syst. 7 (2013) 186-195.

[9] R. Yazicioglu, T. Torfs, J. Penders, I. Romero, H. Kim, P. Merken, B. Gyselinckx, H. Yoo, C. Van Hoof, Ultra-low-power wearable biopotential sensor nodes, in: Annual International Conference of the IEEE Engineering in Medicine and Biology Society, EMBC 2009, 2009, pp. 3205-3208.

[10] A. Bakhshipour, M. Pooyan, H. Mohammadnejad, A. Fallahi, Myocardial ischemia detection with ECG analysis, using wavelet transform and support vector machines, in: 17th Iranian Conference of Biomedical Engineering (ICBME), 2010, pp. 1-4.

[11] W. Deburchgraeve, P. Cherian, M. De Vos, R. Swarte, J. Blok, G. Visser, P. Govaert, S. Van Huffel, Automated neonatal seizure detection mimicking a human observer reading EEG, Clin. Neurophysiol. 119 (2008) 2447-2454.

[12] A. Van de Vel, K. Cuppens, B. Bonroy, M. Milosevic, S. Van Huffel, B. Vanrumste, L.L.B. Ceulemans, Long-term home monitoring of hypermotor seizures by patient-worn accelerometers, Epilepsy Behav. 26 (2013) 118-125.

[13] G. Albright, J. Edie, S. Al-Hallaj, A Comparison of Lead Acid to Lithium-ion in Stationary Storage Applications, white paper, AllCell Technologies LLC, 2012.

[14] X. Cheng, J.Q. Huang, Q. Zhangn, H. Peng, M.Q. Zhao, F. Wei, Aligned carbon nanotube/sulfur composite cathodes with high sulfur content for lithiumsulfur batteries, Nano Energy 4 (2014) 65-72.

[15] T. Horiba, Lithium-ion battery systems, Proc. IEEE 102 (2014) 939-950.

[16] J.Schuder,J.H.Gold,H.E.Stephenson,AninductivelycoupledRFsystemforthe transmission of $1 \mathrm{~kW}$ of power through the skin, IEEE Trans. Biomed. Eng. 18 (1971) 265-273.

[17] J.C. Schuder, Powering an artificial heart: birth of the inductively coupled-radio frequency system in 1960, Artif. Organs 26 (2002) $909-$ 915.

[18] M. Baker, R. Sarpeshkar, Feedback analysis and design of RF power links for low-power bionic systems, IEEE Trans. Biomed. Circuits Syst. 1 (2007) 28-38.

[19] R. Puers, R. Carta, J. Thoné, Wireless power and data transmission strategies for next-generation capsule endoscopes, J. Micromech. Microeng. 21 (2011) 054008.

[20] L.Zhao,C.Foo,K.Tseng,W.Chan,Transcutaneoustransformersinpowersupply system for an artificial heart, in: Proceedings of International Conference on Power Electronic Drives and Energy Systems for Industrial Growth, vol. 1, 1998, pp. 348-352.

[21] B. Lenaerts, R. Puers, Inductive powering of a freely moving system, Sens. Actuators A: Phys. 123-124 (2005) 522-530.

[22] R. Carta, G. Tortora, J. Thoné, B. Lenaerts, P. Valdastri, A. Menciassi, P. Dario, R. Puers, Wireless powering for a self-propelled and steerable endoscopic capsule for stomach inspection, Biosens. Bioelectron. 25 (2009) 845-851.

[23] R. Carta, J. Thoné, R. Puers, A wireless power supply system for robotic capsular endoscopes, Sens. Actuators A: Phys. 162 (2010) $177-$ 183.

[24] P. Jourand, R. Puers, A class-e driven inductive power delivery system covering the complete upper body, Sens. Actuators A: Phys. 183 (2012) 132-139.

[25] R. Carta, M. Sfakiotakis, N. Pateromichelakis, J. Thoné, D. Tsakiris, R. Puers, A multi-coil inductive powering system for an endoscopic capsule with vibratory actuation, Sens. Actuators A: Phys. 172 (2011) 253-258.

[26] ICNIRP, Guidelines for limiting exposure to time-varying electric and magnetic fields, Health Phys. 99 (2010) 818-836.

[27] IEEE, IEEE standard for safety levels with respect to human exposure to radio frequency electromagnetic fields, $3 \mathrm{kHz}$ to $300 \mathrm{GHz}$, $\mathrm{C} 95.1$ $2005,2006$. 


\section{Submitted to Sensors\&Actuators, June 2014}

[28] B. Choi, J. Nho, H. Cha, T. Ahn, B. Choi, Design and implementation of low-profile contactless battery charger using planar printed circuit board windings as energy transfer device, IEEE Trans. Ind. Electron. 51 (2004) 140-147.

[29] C.-H.Hu,C.-M.Chen,Y.-S.Shiao,T.-J.Chan,L.-R.Chen,Developmentofauniversal contactless charger for handheld devices, in: IEEE International Symposium on Industrial Electronics, ISIE 2008, 2008, pp. 99-104.

[30] W. Lim, J. Nho, B. Choi, T. Ahn, Low-profile contactless battery charger using planar printed circuit board windings as energy transfer device, in: IEEE 33rd Annual Power Electronics Specialists Conference, PESC’02, vol. 2, 2002, pp. 579-584.

[31] X. Liu, S. Hui, Equivalent circuit modeling of a multilayer planar winding array structure for use in a universal contactless battery charging platform, IEEE Trans. Power Electron. 22 (2007) 21-29.

[32] K. Hatanaka, F. Sato, H. Matsuki, S. Kikuchi, J. Murakami, M. Kawase, T. Satoh, Power transmission of a desk with a cord-free power supply, IEEE Trans. Magn. 38 (2002) 3329-3331.

[33] N. Shinohara, Power without wires, IEEE Microw. Mag. 12 (2011) S64-S73.

[34] R. Bosshard, J. Muhlethaler, J.W. Kolar, I. Stevanovic, Optimized magnetic design for inductive power transfer coils, in: Proceedings of the 28th Applied Power Electronics Conference and Exposition (APEC 2013), Long Beach, CA, USA, 2013, pp. 1812-1819.

[35] H. De Clercq, R. Puers, A neonatal body sensor network for long-term vital signs acquisition, Proc. Eng. 47 (2012) 981-984.

[36] H. De Clercq, R. Puers, A wireless energy transfer platform, integrated at the bedside, in: 35th Annual International Conference of the IEEE Engineering in Medicine and Biology Society (EMBC), 2013, pp. 1458-1461.

[37] J. Achterberg, E. Lomonova, J. de Boeij, Coil array structures compared for con tactless battery charging platform, IEEE Trans. Magn. 44 (2008) 617-622.

[38] X. Liu, S. Hui, An analysis of a double-layer electromagnetic shield for a universal contactless battery charging platform, in: IEEE 36th Power Electronics Specialists Conference, PESC’05, 2005, pp. 1767-1772.

[39] C.R. Sullivan, Optimal choice for number of strands in a litz-wire transformer winding, IEEE Trans. Power Electron. 14 (1999) $283-291$.

[40] K. Vanschuylenbergh, R. Puers, Inductive Powering: Basic Theory and Application to Biomedical Systems, Springer, New York City, 2009.

[41] ICNIRP. (International Commission on Non-Ionizing Radiation Protection), guidelines for limiting exposure to time-varying electric, magnetic, and electromagnetic fields (up to 300GHz), Health Physics 74 (1998), 494-522.

[42] S.C. Tang, S. Hui, H.-H. Chung, Evaluation of the shielding effects on printed- circuit-board transformers using ferrite plates and copper sheets, IEEE Trans. Power Electron. 17 (2002) 1080-1088.

[43] N. Sokal, A. Sokal, Class E - a new class of high-efficiency tuned single-ended switching power amplifiers, IEEE J. Solid-State Circuits 10 (1975) 168-176.

[44] M. Meyerhoff, Understanding newborn characteristics and development, Online: http://lifestyle.howstuffworks.com/family/parenting/babies/ understanding-newborn-characteristics-and-development-ga.htm, 2006 (accessed: 16.06.14).

[45] G. Kendir, W. Liu, G. Wang, M. Sivaprakasam, R. Bashirullah, M. Humayun, J. Weiland, An optimal design methodology for inductive power link with class-E amplifier, IEEE Trans. Circuits Syst. I, Regul. Papers 52 (2005) 857-866.

[46] J. Ziegler, N. Nichols, Optimum settings for automatic controllers, Trans. ASME 64 (1942) 759-768.

[47] B. Lenaerts, R. Puers, Omnidirectional Inductive Powering for Biomedical Implants, Springer, New York City, 2009. 


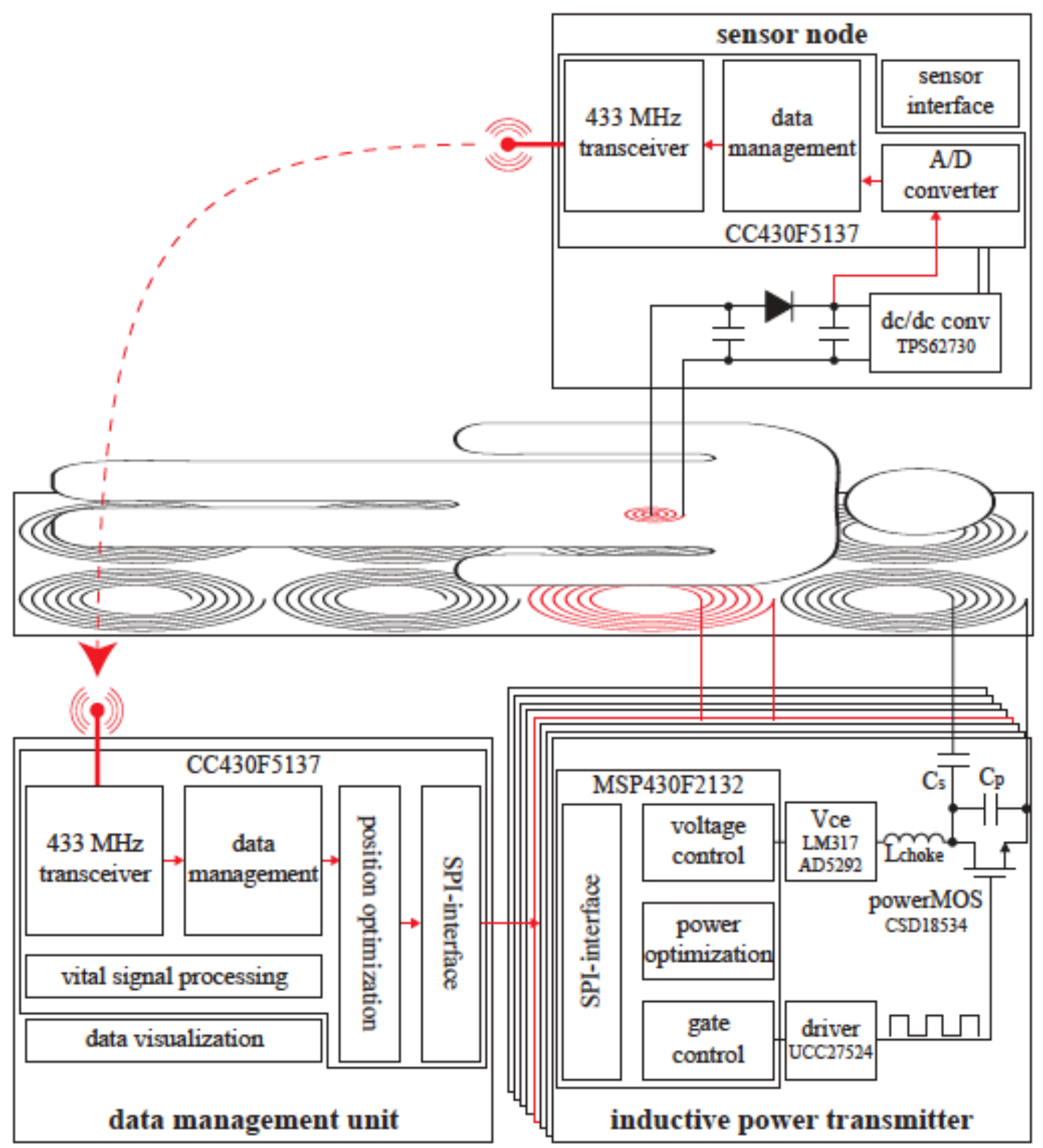

Figure 1: A block-level schematic of the contactless energy transfer platform, integrated at the bedside. The power feedback loop is marked in red. 


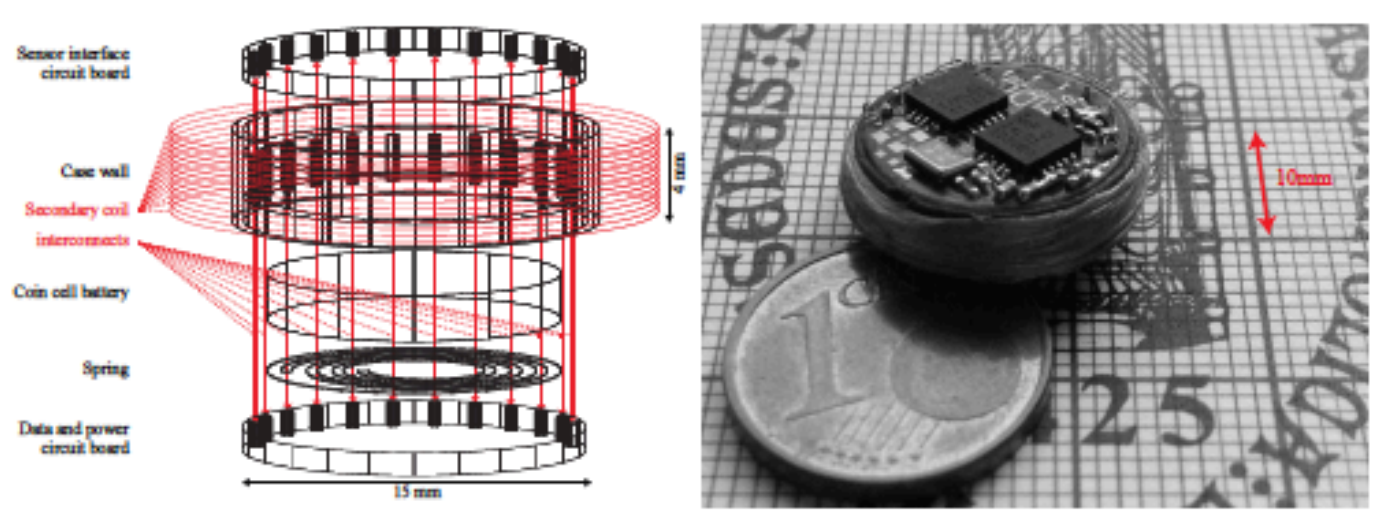

Figure 2: Construction of the remote wearable sensor node showing the different components of the sensor node (left) and an assembled prototype (right).

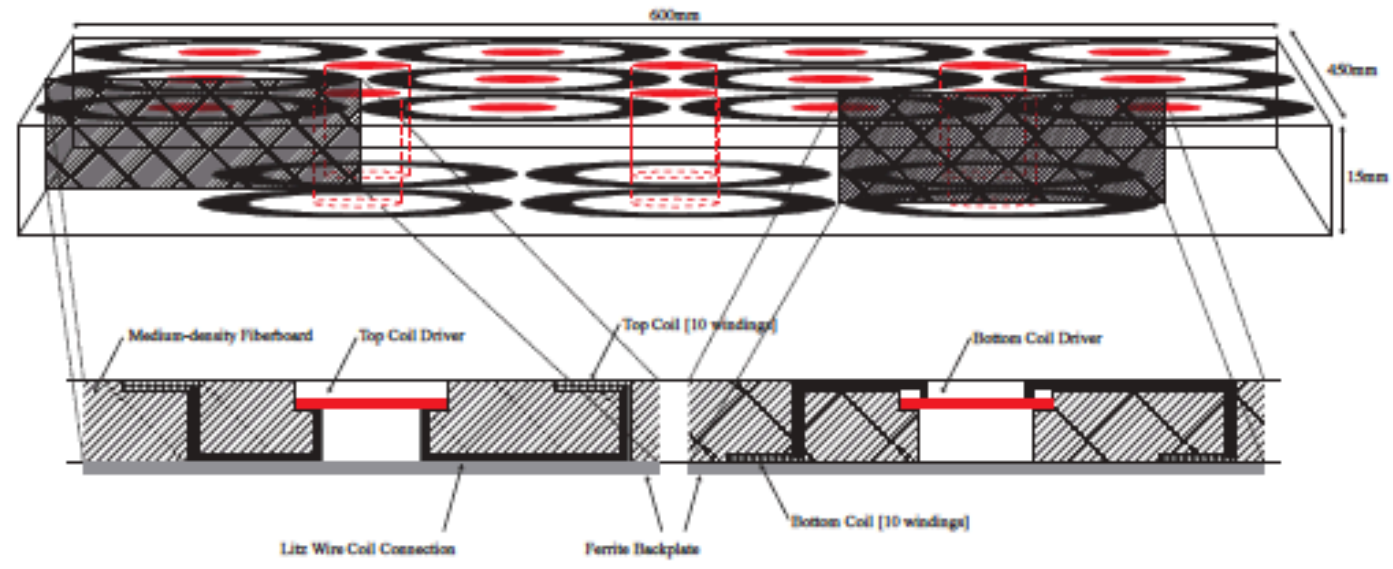

Figure 3: The matrix of eighteen identical pancake base coils in a two-layer configuration. The circular printed circuit boards are coloured in red, the Litz wire base coils are colored in black. 


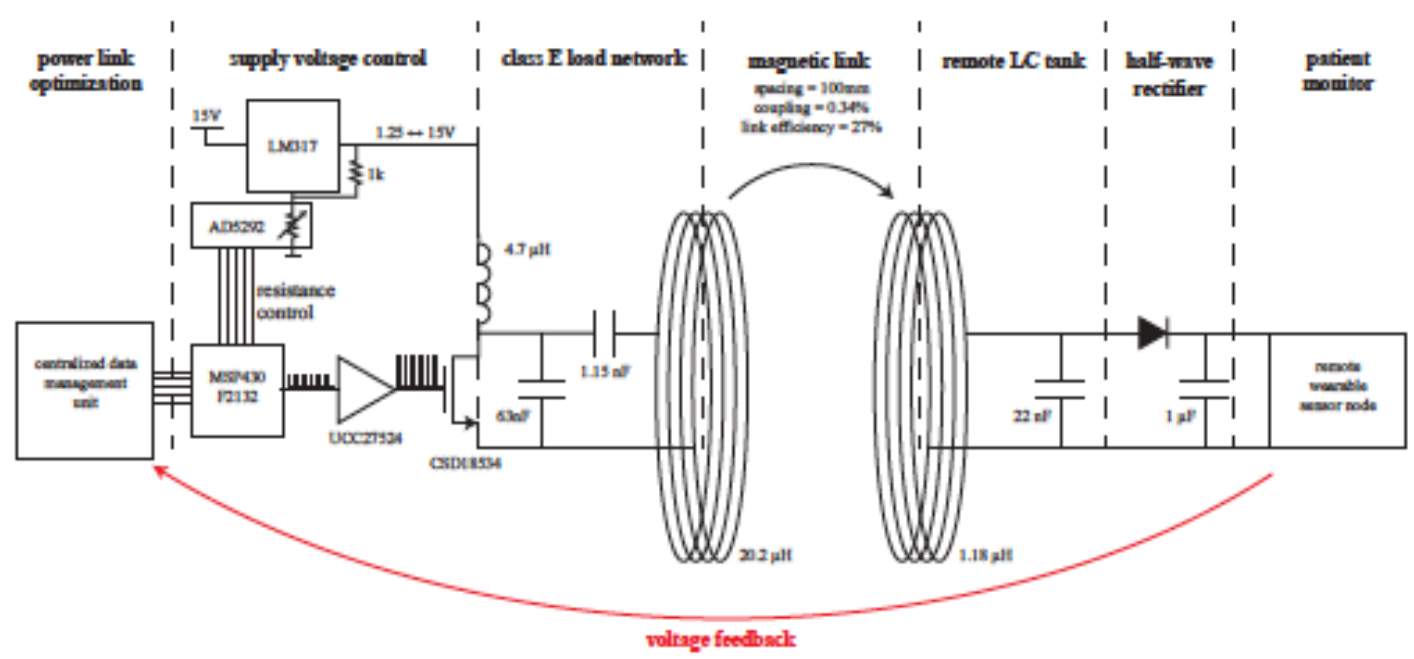

Figure 4: The circuit-level schematic of the magnetic power link.

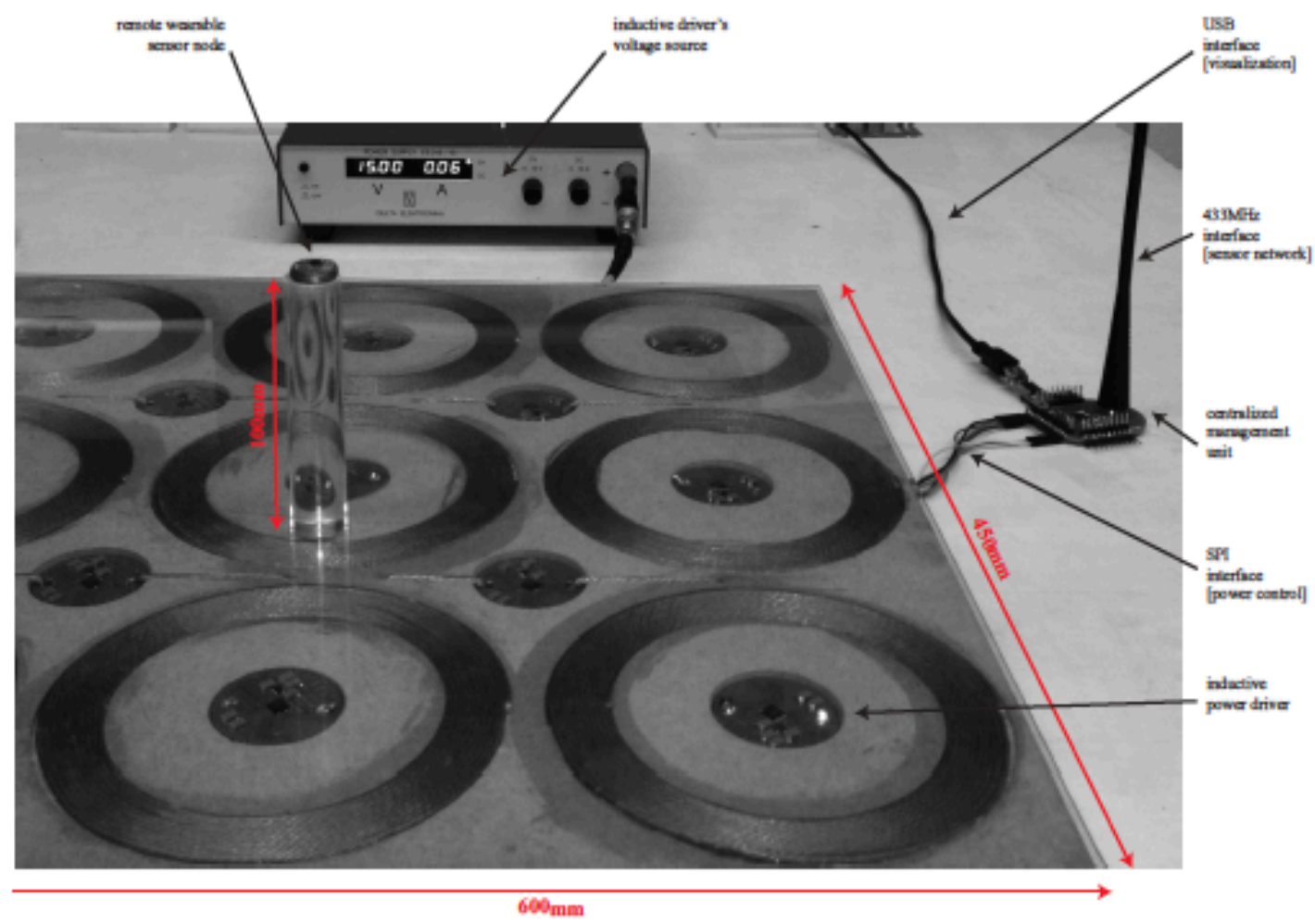

Figure 5: Photograph of the contactless energy transfer platform, containing the remote wearable sensor node the inductive power transmitter and the matrix of identical base coils integrated at the bedside. 
Driver's input (Vgs): square wave with a duty cycle of $25 \%$

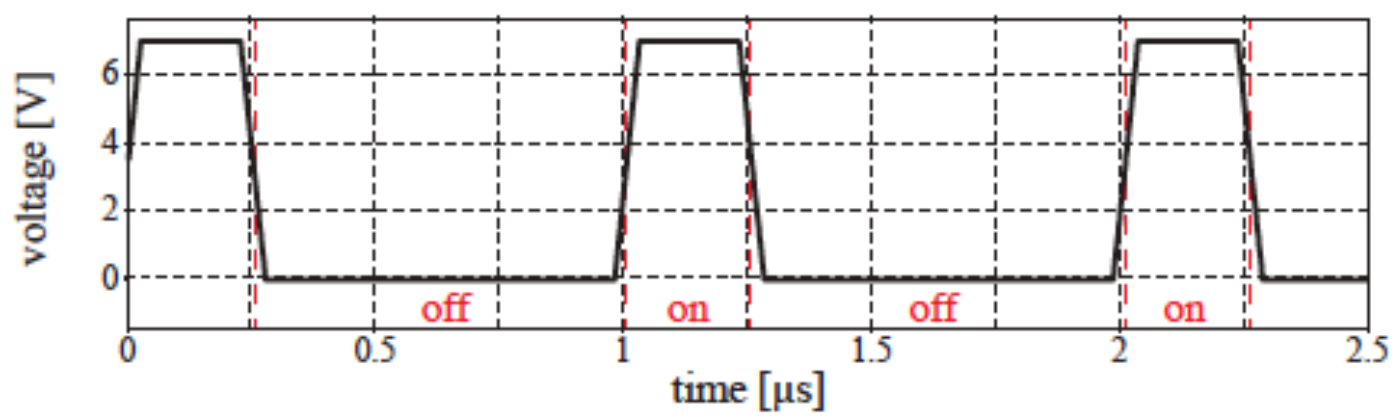

Class E voltage signal (Vds)

Class E current signal (Ids)
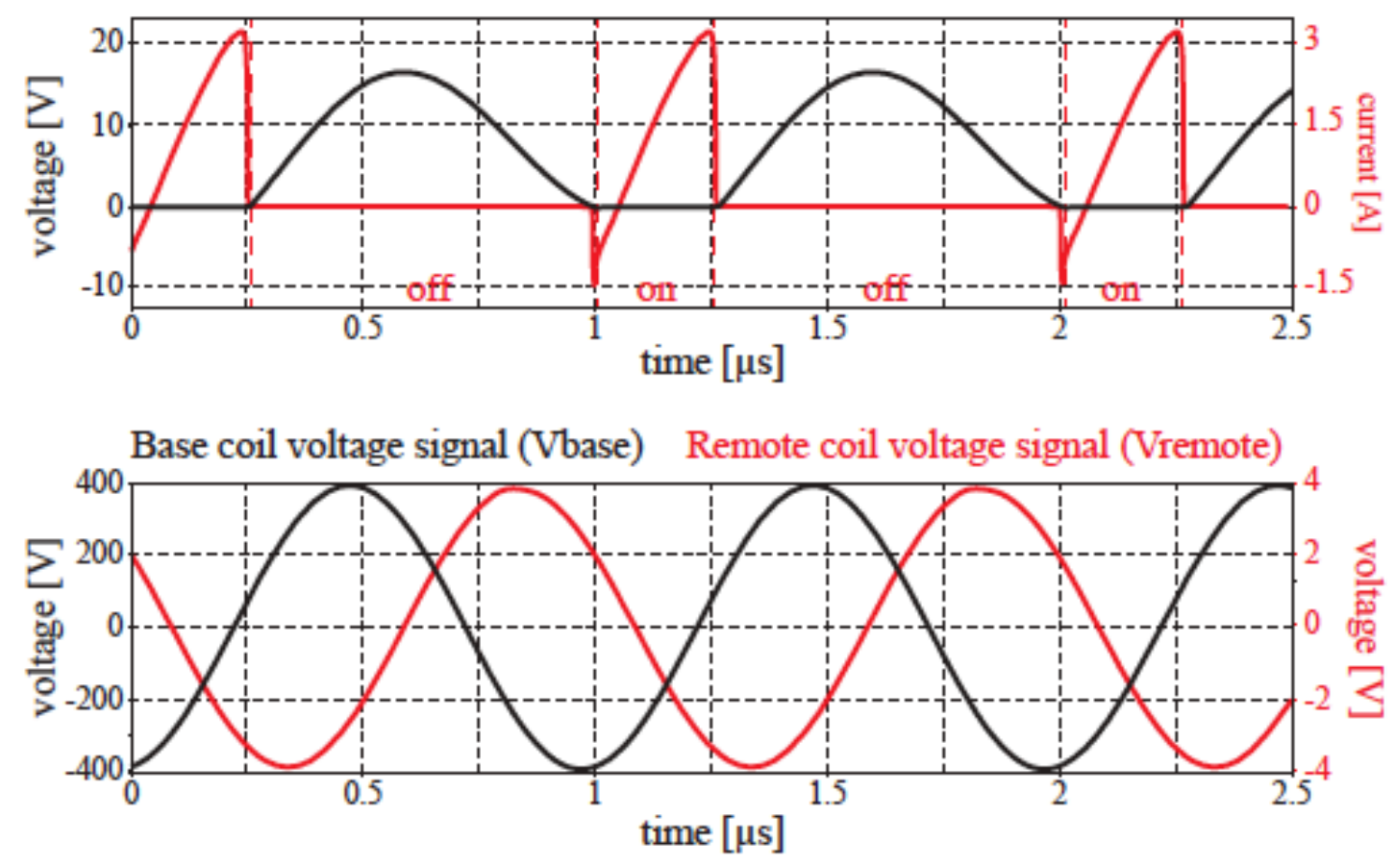

Figure 6: Spice simulations of a dedicated Class E driver of an inter-coil distance of $220 \mathrm{~mm}$. The gate signal with a duty cycle of $25 \%$ (top graph), The voltage over and the current through the MOSFET channel (middle) and the voltages over the base and remote coil. 
Submitted to Sensors\&Actuators, June 2014

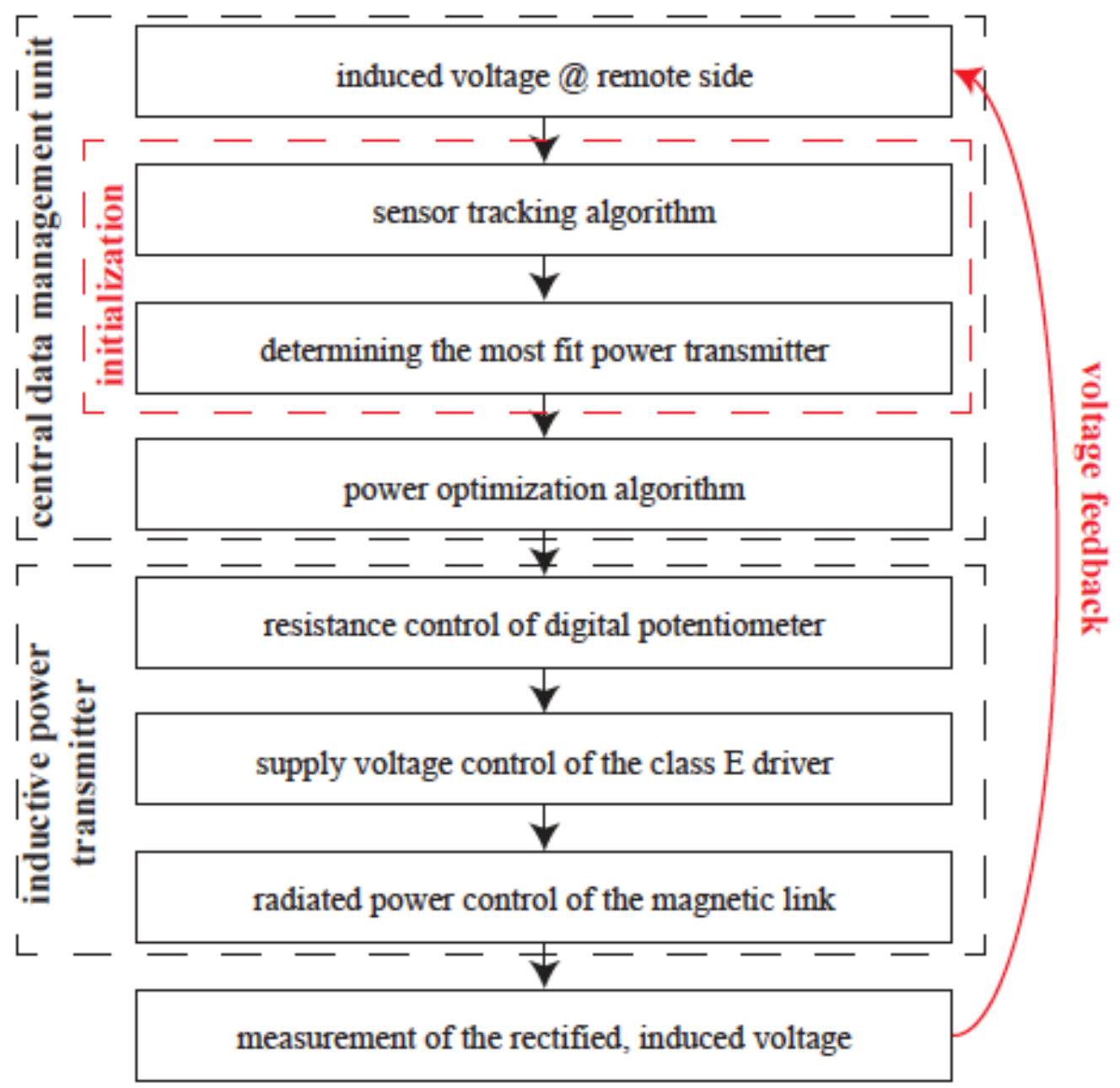

Figure 7: The power optimisation strategy for inductive powering at the bedside. The sensor tracking algorithm is only carried out during the initialization step. 
Driver's input (Vgs): square wave with a duty cycle of $25 \%$

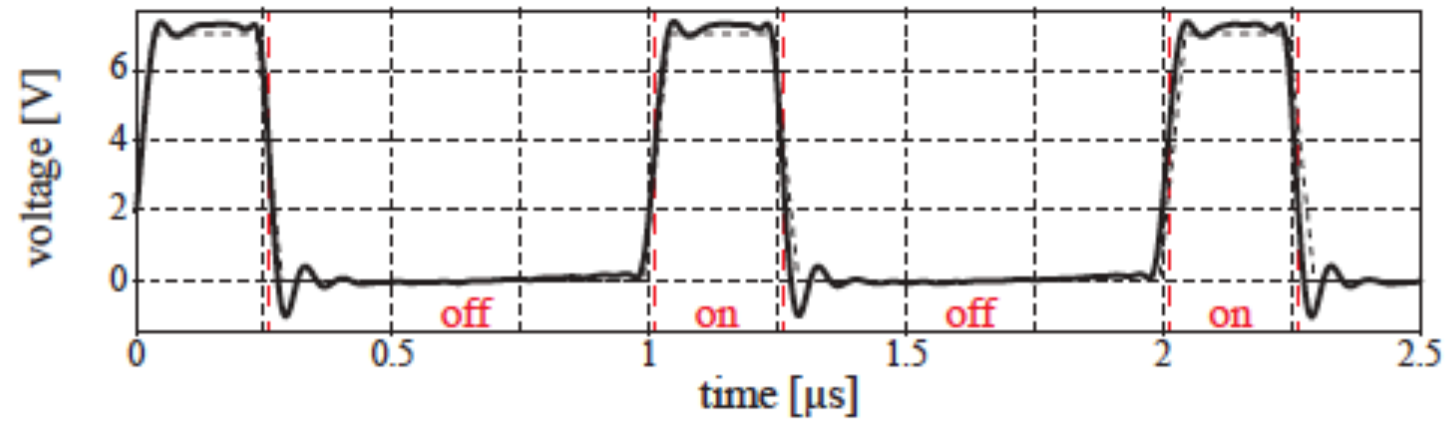

\section{Class E voltage signal (Vds)}
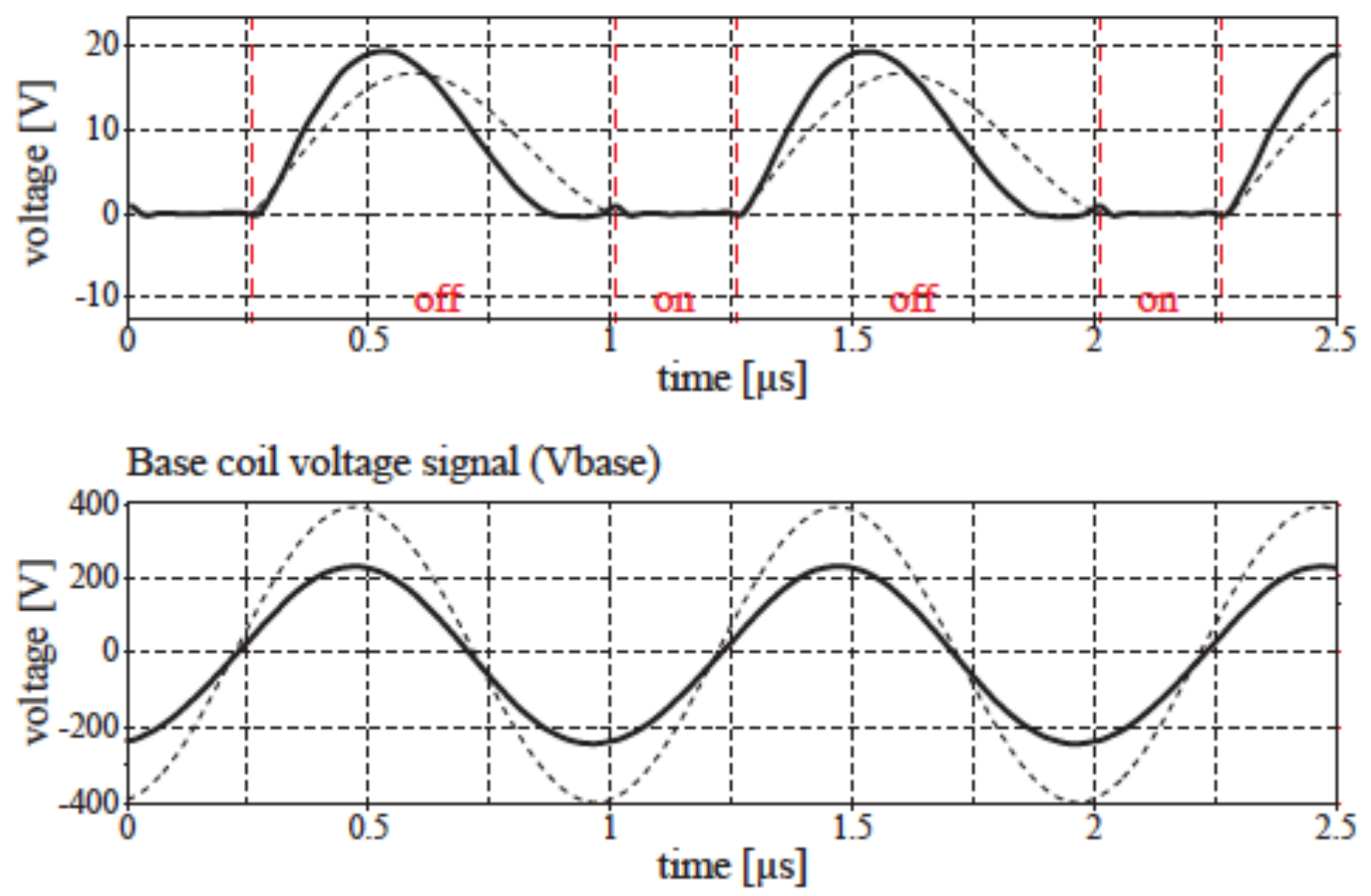

Figure 8: Measurement results of the class E power driver. The gate signal with a duty cycle of $25 \%$ (top), the voltage over the power transistor (middle) and the voltage over the subjected base coil (bottom). The corresponding simulation results are added as a comparison (dashed lines) 
Supply voltage with power feedback Supply voltage without feedback
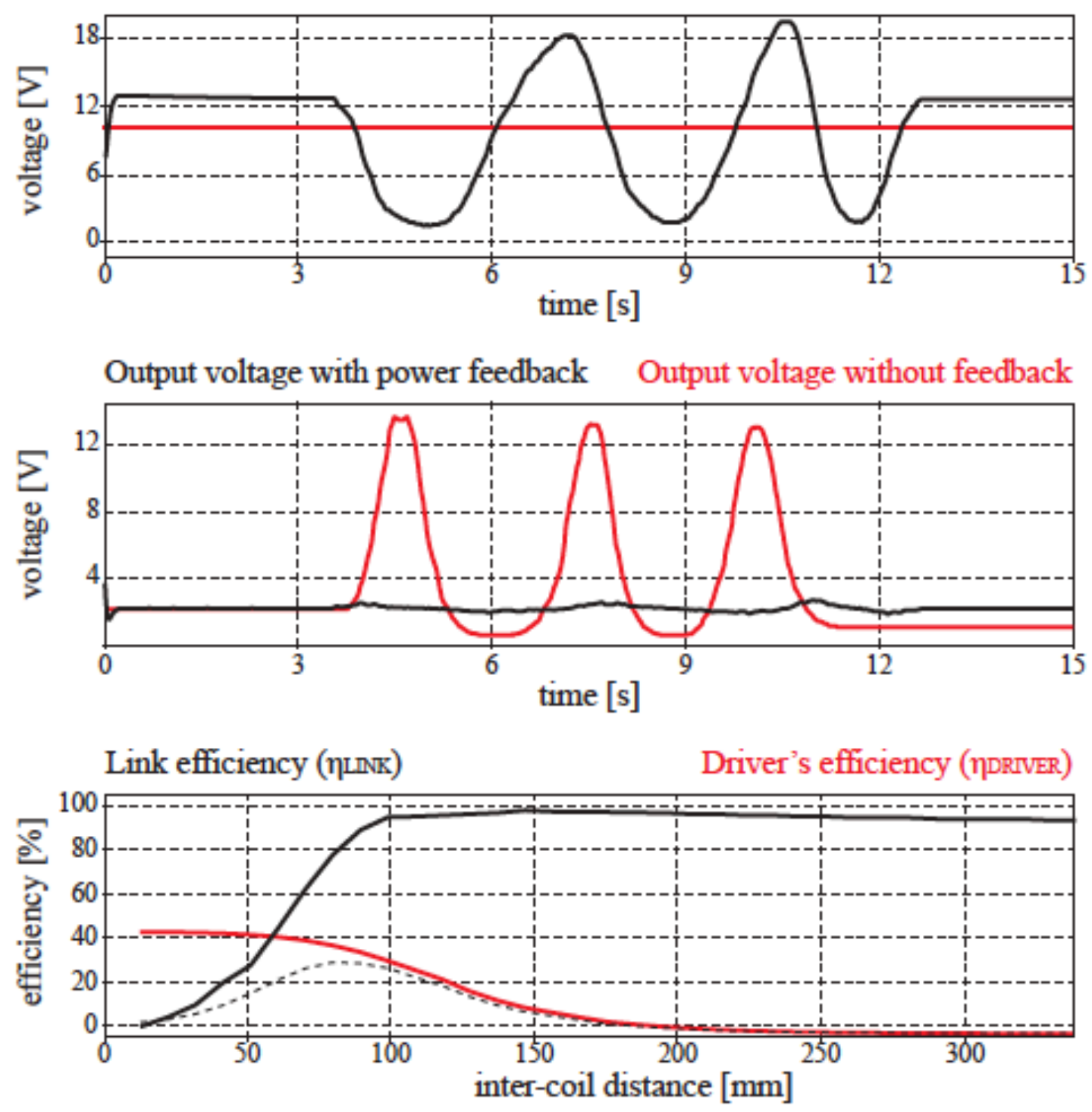

Figure 9: Measurement results of the online power optimization algorithm, compared to the classical approach (marked in red). 